\title{
Six-month exclusive breastfeeding and the impact of COVID-19 on breastfeeding practice in Hong Kong: A mixed-methods study
}

\section{John Kwan ( $\sim$ johnkwan@hku.hk)}

The University of Hong Kong Department of Paediatrics and Adolescent Medicine https://orcid.org/0000-0002-9616-9657

Jimsyn Jia

The University of Hong Kong Department of Paediatrics and Adolescent Medicine

Ka-man Yip

The University of Hong Kong Department of Paediatrics and Adolescent Medicine

\section{Hung-kwan So}

The University of Hong Kong Department of Paediatrics and Adolescent Medicine

\section{Sophie SF Leung}

Chinese University of Hong Kong Department of Paediatrics

\section{Patrick Ip}

The University of Hong Kong Department of Paediatrics and Adolescent Medicine

\section{Wilfred HS Wong}

The University of Hong Kong Department of Paediatrics and Adolescent Medicine

\section{Research Article}

Keywords: Breastfeeding, exclusive breastfeeding, COVID-19, family support, public hospital, private hospital, Baby-Friendly Hospital Initiative, antenatal class, knowledge, Hong Kong

Posted Date: July 8th, 2021

DOl: https://doi.org/10.21203/rs.3.rs-680817/v1

License: (c) (i) This work is licensed under a Creative Commons Attribution 4.0 International License. Read Full License 


\section{Abstract \\ Background}

The World Health Organization recommends exclusive breastfeeding (EBF) for an infant's first six months. Therefore, various policies were implemented to support breastfeeding in Hong Kong in the past decade. Meanwhile, the COVID-19 pandemic brought new challenges and opportunities to breastfeeding practices. This study aimed to identify the associated factors of six-month EBF, and to evaluate the impact of COVID-19 on breastfeeding practice.

\section{Methods}

This study was conducted using a mixed-methods approach. An electronic questionnaire was distributed to members of breastfeeding or parenting groups who have had breastfeeding experience in the past 10 years. Exploratory factor analysis with principal components analysis was used to identify the factors representing breastfeeding support from family and friends. Stepwise binary logistic regression analyses with forward (conditional) selection were conducted to examine factors associated with six-month EBF both in general and during the pandemic period. A thematic analysis was conducted using both deductive and inductive approaches.

\section{Results}

The study included 793 participants. Giving birth in a public hospital (AOR 2.015, 95\% Cl 1.440 to 2.821, $\mathrm{p}<0.001$ ) and breastfeeding support from family and friends (AOR 1.309, 95\% $\mathrm{Cl} 1.102$ to $1.555, \mathrm{p}=$ 0.002) were significantly associated with six-month EBF even during COVID-19. Our qualitative results indicated a high prevalence of breastfeeding problems in both public and private maternity wards, suggesting that neonatal support for breastfeeding in Hong Kong were generally insufficient and ineffective.

\section{Conclusions}

Giving birth in a public hospital and having breastfeeding support from family and friends were associated with six-month EBF. Furthermore, COVID-19 in Hong Kong had an overall positive impact on six-month EBF. Further studies should investigate the impact of hospital practice and the COVID-19 pandemic on breastfeeding behaviours.

\section{Background}

The World Health Organization (WHO) recommends exclusive breastfeeding (EBF), the exclusive consumption of breast milk with no additional solids or liquids, for an infant's first six months; after 
which breastfeeding is accompanied with complementary foods for up to two years or beyond [1]. Welldocumented child health benefits of such a breastfeeding practice include optimal growth, intelligence, protection against infections, and reduced risks of chronic diseases during adulthood [2]. However, as of 2018, the global EBF rate for children aged below six months was $41 \%$, which fell short of the $70 \%$ target of the WHO 2030 global nutrition goals [3].

In Hong Kong, various policies have been developed to support breastfeeding over the past decade. In 2010, public hospitals in Hong Kong stopped accepting free breast milk substitutes from manufacturers [4]; such a pivotal policy reduced the availability of formula and encouraged breastfeeding practices amongst new breastfeeding mothers. Meanwhile, the Hospital Authority is committed to supporting all eight public hospitals with obstetric wards to comply with the WHO-launched Baby-Friendly Hospital Initiative (BFHI) and implement the "Ten Steps to Successful Breastfeeding". In 2017, the Food and Health Bureau issued the Hong Kong Code of Marketing of Formula Milk and Related Products, and Food Products for Infants \& Young Children to protect breastfeeding in response to the aggressive marketing of formula milk in Hong Kong [5]. Recently, the Discrimination Legislation (Miscellaneous Amendments) Ordinance 2020 came into force to protect breastfeeding women from direct or indirect discrimination, and the Employment (Amendment) Ordinance 2020 was established to extend statutory maternity leave from 10 weeks to 14 weeks [6, 7].

Consequently, the breastfeeding initiation rate of Hong Kong increased significantly from $76.7 \%(2010)$ to 87.2\% (2019), keeping up with other Asian jurisdictions such as the Republic of Korea $(90.2 \%)[8,9]$. Despite so, the EBF rate at the first month remained low at $32.6 \%$ in Hong Kong, while EBF rate at the sixth month further dropped to $26.3 \%$ [10], outperforming the Republic of Korea (2.3\%), but underperforming Japan (37.4\%) and Taiwan (46.2\%) [9-12]. Moreover, early breastfeeding cessation remains common in Hong Kong. Between the first and sixth months, 19.3\% of EBF mothers discontinued EBF [10]. To raise the EBF rate, it is important to identify factors associated with EBF in order to support sustainable breastfeeding practices. Previous studies have identified the barriers of EBF, including the lack of knowledge about the superior health benefits of breastfeeding, inadequate breastfeeding skills, sociocultural norms and beliefs, poor family support, embarrassment of breastfeeding in public places, and the lack of workplace support [13].

During the COVID-19 pandemic which first struck Hong Kong in January 2020, the government and different organisations implemented work-from-home arrangements, while breastfeeding coaching services in maternal and child health centres (MCHCs) were temporarily suspended [14, 15]. Although it is believed that the breastfeeding rate would be affected by these measures, limited studies have been conducted to examine the impact of COVID-19 on breastfeeding internationally, while none were conducted in Hong Kong [16].

Therefore, this study aimed to identify factors associated with the practice of EBF for at least six months since birth (hereafter referred to as "six-month EBF"), with a focus on the barriers and facilitators of EBF, as well as the perception of Hong Kong women who have had experience breastfeeding within the past 
10 years. Furthermore, this study aimed to evaluate the impact of COVID-19 on EBF and investigate the unique opportunities and challenges for breastfeeding brought by COVID-19. In addition, this paper shall provide recommendations for encouraging EBF.

\section{Methods}

Study design and participants

This study was conducted using a mixed-methods research design comprising both quantitative and qualitative analytical approaches. A purposive sampling approach was adopted to target mothers with breastfeeding experience, breastfeeding determination, and experience with a wide range of breastfeeding services provided by different health care providers, organisations, and professionals. Hence, only members of breastfeeding or parenting groups were invited to participate in the study; such support groups include the Hong Kong Breastfeeding Mothers' Association (HKBFMA) and La Leche League Hong Kong (LLLHK). Specifically, the inclusion criteria were Hong Kong mothers with breastfeeding experience in the past 10 years, so as to evaluate the multitude of breastfeeding policies that were put forth since 2010 .

Data collection

An electronic questionnaire was developed to investigate the breastfeeding practice and experience of Hong Kong mothers [see Additional file 1]. The content of the questionnaire was based on the suggestions from Tarrant et al [17], while questions regarding breastfeeding knowledge and the perceived support from workplace and public premises were partially adopted from the Baby Friendly Hospital Initiative Hong Kong Association (BFHIHKA) and the Department of Health with modifications [18, 19]. Question types included Likert scale, polar, categorical, and open-ended questions. An independent panel of six members, consisting of registered nurses, International Board Certified Lactation Consultants (IBCLCs), and paediatricians, was consulted during the questionnaire development process. A pilot trial was conducted with the panel to test the questionnaire. The questionnaire was distributed on the Facebook platform of HKBFMA and LLLHK. Responses were collected from 1st March 2021 to 7th April 2021.

Analysis

\section{Statistical analysis}

Descriptive statistics were used to examine the characteristics of all participants. Examined characteristics include maternal age, maternal education level, maternal employment status, monthly total household income, the size of residential property, number of people in the household, and birth hospital (public or private). In particular, for participants whose child was six months or older, chi-squared test and Fisher's exact test were used to compare their categorical variables in relation to their practice of six-month EBF. 
Exploratory factor analysis (EFA) with principal components analysis was used to identify the factor representing the support for breastfeeding from family and friends, which was later included in binary logistic regression analysis to examine its association with successful six-month EBF amongst participants whose child was six months or older. An eigenvalue of $>1$ was used to extract the factor. Missing values were replaced with the mean, and variables with factor loading greater than or equal to 0.4 were used for analysis [20].

Stepwise binary logistic regression analysis with forward (conditional) selection was used to determine factors associated with successful six-month EBF in participants whose child was six months or older. The following variables were included: breastfeeding support from family and friends, breastfeeding knowledge score, and birth hospital (public or private), adjusted for household income per capita, maternal age, maternal education level, maternal employment status, and size of residential property.

To investigate the impact of the COVID-19 pandemic on successful six-month EBF, stepwise binary logistic regression analysis with forward (conditional) selection was performed amongst participants whose child was six to twelve months old. The following variables were included: breastfeeding support from family and friends, breastfeeding knowledge score, birth hospital (public or private), and self-rated impact of COVID-19 on breastfeeding, adjusted for household income per capita, maternal age, maternal education level, maternal employment status, and size of residential property.

Two-sided p-value of less than 0.05 was considered statistically significant. All analyses were performed using IBM SPSS Statistics for Macintosh, version 26.0 (IBM Corp., Armonk, NY, USA).

\section{Qualitative analysis}

Qualitative analysis was performed for all participants. Multiple choice options and open-ended responses in the questionnaire were respectively coded using deductive and inductive approaches to analyse participants' (a) reasons for EBF cessation, (b) opinions on antenatal classes, (c) breastfeeding challenges encountered in maternity wards, (d) opinions on the service provided by different health organisations and professionals, and (e) opinions on the impact of COVID on breastfeeding.

Using a deductive approach, initial codes regarding participants' opinions on $\mathrm{MCHC}$ services and parts (a), (c), and (e) were designed and presented as multiple choice options for participants to select. Meanwhile, an inductive coding method was adopted to generate codes from additional open-ended responses regarding parts (a) to (e); induced codes were then categorised as appropriate.

A thematic analysis was subsequently performed based on such categories. In addition, responses regarding participants' experience in public and private hospital maternity wards and breastfeeding clinics were separately analysed, so as to contrast the breastfeeding support offered by public and private hospitals. Chi-square tests were further performed to compare the deduced codes of public and private hospitals. 


\section{Results}

A total of 880 responses were made, of which 793 (90.1\%) were complete and met the inclusion criteria. Of the 656 participants whose child was six months or older, 286 (43.6\%) had practiced six-month EBF, while $370(56.4 \%)$ had not.

Characteristics and breastfeeding pattern of participants

Table 1 shows the characteristics and breastfeeding pattern of all participants. The majority $(77.4 \%)$ of participants were between 31 and 40 years of age, and $82.1 \%$ had post-secondary degrees. There were $57.4 \%$ participants employed full time, $9.2 \%$ employed part time, $6.9 \%$ on maternity leave, and $26.5 \%$ were housewives. Amongst all participants, $60.2 \%$ gave birth in a public hospital. There were more 4-person households $(P=0.008)$ and a greater proportion of public hospital births $(P<0.001)$ in participants who had practiced six-month EBF. 
Table 1

Characteristics and breastfeeding pattern of participants

\section{Characteristic}

Maternal age

$21-30$

8.6

12.4

77.4

83.2

$14.0 \quad 4.4$

$\geq 41$

Maternal education

Secondary or below

Post-secondary: Diploma

/ certificate

Post-secondary: Sub-

degree course

Post-secondary: Degree course

\section{Maternal employment} status

Employed (full time)

$57.4 \quad 36.5$

59.8

10.1

26.9

3.1

On maternity leave

9.2

4.4

Monthly total household income (HKD)

$<=29999$

30000-49999

50000-69999

70000-89999
$6.9 \quad 28.5$

30.7

7.8

9.8

$14.1 \quad 15.0$

26.5

19.8

19.8

$15.5 \quad 12.0$ EBF
8.0

8.4

2.1

81.5

$\begin{array}{ll}7.0 & 8.4\end{array}$

74.5

77.6

18.5

14.1

8.4
77.6
4.1

7.8

7.8

6.2

2.2
0.270

Not sixmonth EBF Pvalue* $(n=370)$

$\%$

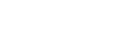

more

0.757 


\begin{tabular}{|c|c|c|c|c|c|}
\hline \multirow[t]{2}{*}{ Characteristic } & $\begin{array}{l}\text { Total } \\
(n= \\
793)\end{array}$ & $\begin{array}{l}\text { Children aged less than } 6 \\
\text { months }(n=137) \\
\%\end{array}$ & \multicolumn{3}{|c|}{ Children aged 6 months or more } \\
\hline & $\begin{array}{l}(n= \\
793) \\
\%\end{array}$ & $\%$ & $\begin{array}{l}\text { Six- } \\
\text { month } \\
\text { EBF } \\
(n=286) \\
\%\end{array}$ & $\begin{array}{l}\text { Not six- } \\
\text { month EBF } \\
(n=370) \\
\%\end{array}$ & $\begin{array}{l}\text { P- } \\
\text { value* }\end{array}$ \\
\hline $90000-109999$ & 15.0 & 15.8 & 16.1 & 13.9 & \\
\hline$>=110000$ & 27.6 & 24.1 & 28.3 & 28.3 & \\
\hline $\begin{array}{l}\text { Size of residential } \\
\text { property (sq. ft.) }\end{array}$ & & & & & 0.091 \\
\hline$<=400$ & 17.3 & 16.3 & 16.3 & 18.5 & \\
\hline $400-599$ & 23.4 & 29.6 & 18.4 & 25.0 & \\
\hline $600-799$ & 24.4 & 22.2 & 25.4 & 24.5 & \\
\hline$>=800$ & 34.9 & 31.9 & 39.9 & 32.1 & \\
\hline $\begin{array}{l}\text { Number of people in the } \\
\text { household }\end{array}$ & & & & & 0.008 \\
\hline 2 & 0.5 & 1.5 & 0.0 & 0.5 & \\
\hline 3 & 34.6 & 34.6 & 28.3 & 39.6 & \\
\hline 4 & 39.5 & 35.3 & 45.2 & 36.6 & \\
\hline$>=5$ & 25.4 & 28.7 & 26.5 & 23.3 & \\
\hline Birth hospital & & & & & $<.001$ \\
\hline Public hospital & 60.2 & 53.3 & 69.9 & 55.1 & \\
\hline Private hospital & 39.8 & 46.7 & 30.1 & 44.9 & \\
\hline
\end{tabular}

Exploratory factor analysis

Table 2 shows the items and factor loadings for the identified factor by EFA. Bartlett's test of sphericity rejected the null hypothesis of an identity matrix $\left(X^{2}=305.7, P<0.001\right)$. The total variance explained by this one-factor model was $60.9 \%$. The initial eigenvalue of the factor was 1.828 . The factor contained items on spouse's, parents', and friends' support towards breastfeeding, and was labelled 'breastfeeding support from family and friends". 
Table 2

Summary of items and factor loadings for the identified factor

\section{Item}

Factor loading

Factor: Breastfeeding support from family and friends

(24) Spouse is supportive of breastfeeding.

0.755

(25) Parents are supportive of breastfeeding.

0.832

(26) Friends are supportive of breastfeeding.

0.752

Factors associated with successful six-month EBF

The binary logistic regression models are shown in Tables 3 and 4. After adjustment by household income per capita, maternal age, maternal education level, maternal employment status, and size of residential property, two factors were significantly associated with successful six-month EBF, namely giving birth in a public hospital (AOR 2.015, 95\% Cl 1.440 to $2.821, P<0.001$ ) and breastfeeding support from family and friends (AOR $1.309,95 \% \mathrm{Cl} 1.102$ to $1.555, P=0.002$ ). During COVID-19, three factors were significantly associated with successful six-month EBF, namely giving birth in a public hospital (AOR $2.784,95 \% \mathrm{Cl} 1.657$ to $4.677, P<0.001$ ), breastfeeding support from family and friends (AOR $1.379,95 \%$ Cl 1.032 to $1.842, P=0.030$ ), and self-rated impact of COVID-19 on breastfeeding (AOR $1.118,95 \% \mathrm{Cl}$ 1.005 to $1.243, P=0.040)$.

Table 3

Factors associated with successful six-month EBF in binary logistic regression model

\begin{tabular}{|lllll|}
\hline & AOR & SE & $\begin{array}{l}\text { P- } \\
\text { value }\end{array}$ & $\begin{array}{l}95 \% \text { Cl for AOR (lower to } \\
\text { upper) }\end{array}$ \\
\hline Birth hospital & & & & \\
\hline Public & 2.015 & 0.172 & $\begin{array}{l}< \\
0.001\end{array}$ & 1.440 to 2.821 \\
\hline $\begin{array}{l}\text { Private } \\
\begin{array}{l}\text { Breastfeeding support from family and } \\
\text { friends }\end{array}\end{array}$ & 1.309 & 0.088 & 0.002 & 1.102 to 1.555 \\
\hline
\end{tabular}

*Adjusted for household income per capita, maternal age, maternal education level, maternal employment status, size of residential property. 
Table 4

Factors associated with successful six-month EBF during COVID-19 in binary logistic regression model

\begin{tabular}{|llllll|}
\hline & AOR & SE & $\begin{array}{l}\text { P- } \\
\text { value }\end{array}$ & $\begin{array}{l}95 \% \text { Cl AOR (lower to } \\
\text { upper) }\end{array}$ \\
\hline Birth hospital & & & & & \\
\hline Public & 2.784 & 0.265 & $<$ & 1.657 to 4.677 \\
\hline Private & Reference & & & \\
\hline $\begin{array}{l}\text { Breastfeeding support from family and } \\
\text { friends }\end{array}$ & 1.379 & 0.148 & 0.030 & 1.032 to 1.842 \\
\hline $\begin{array}{l}\text { Self-rated impact of COVID-19 on } \\
\text { breastfeeding }\end{array}$ & 1.118 & 0.054 & 0.040 & 1.005 to 1.243 \\
\hline
\end{tabular}

*Adjusted for household income per capita, maternal age, maternal education level, maternal employment status, size of residential property.

Qualitative analysis

\section{Reasons for EBF cessation}

There were 391 participants who provided reasons for stopping EBF. Common deduced reasons include natural weaning (47.1\%), perceived insufficient milk supply (PIMS) (39.4\%), busyness of life (24.0\%), and returning to work from maternity leave (21.7\%). In addition, 23 valid open-ended responses were obtained, of which nine attributed EBF cessation to "maternal mental distress".

\section{Opinions on antenatal classes}

Of the 577 participants who had attended antenatal classes, $37.4 \%$ found them unhelpful in guiding breastfeeding, $36.7 \%$ found them helpful, and $25.8 \%$ remained neutral. In addition, 317 valid open-ended responses were collected, from which conflicting themes were induced.

Regarding pessimistic themes, 92 participants indicated that "breastfeeding can only be effectively learnt postnatally and practically, instead of antenatally", 75 indicated that "information offered in antenatal classes are incomprehensive, theoretical, understated, or generic", 38 indicated that "antenatal classes could not address the unique breastfeeding challenges encountered by each mother-infant pair", and 32 indicated "an insufficient time or emphasis on breastfeeding issues during their antenatal classes". In addition, four participants indicated that "most antenatal classes were cancelled or taught online during COVID-19".

In contrast, 58 optimistic responses indicated that antenatal classes "equip mothers with basic but helpful breastfeeding information which offer them a head start". 
Around $79 \%$ of participants who gave birth in public hospitals encountered some form of breastfeeding problems, while nearly $83 \%$ of participants who gave birth in private hospitals did so. The deduced breastfeeding problems encountered by participants are summarised in Table 5.

Table 5

Difficulties encountered in maternity wards

\begin{tabular}{|c|c|c|c|}
\hline & $\begin{array}{l}\text { Public } \\
(n= \\
477)\end{array}$ & $\begin{array}{l}\text { Private } \\
(n= \\
316)\end{array}$ & $\begin{array}{l}\mathrm{P} \text { - } \\
\text { value }\end{array}$ \\
\hline & $\%$ & $\%$ & \\
\hline Latching issues & 55.1 & 52.2 & 0.093 \\
\hline $\begin{array}{l}\text { Need to supplement with other milk sources due to low breast milk } \\
\text { supply }\end{array}$ & 27.5 & 40.2 & $<.001$ \\
\hline $\begin{array}{l}\text { Unable to initiate breastfeeding within } 30 \text { minutes after delivery / } \\
\text { becoming responsive }\end{array}$ & 27.5 & 35.1 & 0.044 \\
\hline Nurses were too busy to offer effective breastfeeding assistance & 28.9 & 15.2 & $<001$ \\
\hline Midwives were too busy to offer effective breastfeeding assistance & 20.3 & 7.6 & $<.001$ \\
\hline None & 20.8 & 17.4 & 0.243 \\
\hline
\end{tabular}

Latching problem was the most common breastfeeding problem encountered in both public maternity wards $(55.1 \%)$ and private maternity wards $(52.2 \%)$; the prevalence of latching problem in public and private maternity wards showed no significant difference $(P=0.09)$. However, compared to public hospital participants, a significantly greater proportion of private hospital participants supplemented with other milk sources due to PIMS $(40.2 \%>27.5 \%, P<0.001)$ and failed to initiate breastfeeding within 30 minutes after delivery or being responsive $(35.1 \%>27.5 \%, P=0.04)$. Meanwhile, compared to private hospital participants, a significantly greater proportion of public hospital participants did not receive effective breastfeeding assistance due to the busyness of nurses $(28.9 \%>15.2 \%, P<0.001)$ and midwives $(20.3 \%>7.6 \%, P<0.001)$.

Of the 60 public hospital participants who provided valid open-ended responses, 17 received "poor breastfeeding support in special care baby units (SCBUs) and neonatal intensive care units (NICUs)". Meanwhile, of the 21 private hospital participants who provided valid open-ended responses, four complained that "nurses inappropriately recommended formula feeding as first-line solution for all breastfeeding issues", three complained that "midwives or nurses offered conflicting and incomprehensive breastfeeding advice", and three indicated that "infants were unnecessarily separated from mothers due to non-medical reasons". 


\section{Opinions on different health organisations and professionals}

Of all participants who sought breastfeeding coaching services due to breastfeeding difficulties $(n=503)$, most sought help from MCHCs (58.3\%), IBCLCs (32.4\%), breastfeeding support organisations $(29.2 \%)$, and confinement nannies (23.3\%).

Open-ended responses indicated that IBCLCs, private nurses, and certified milk enhancing workers were available for "personalised support" and "home visits", while private breastfeeding clinics, private doctors, and confinement nannies received mixed open-ended responses regarding their "supportiveness", "breastfeeding knowledge", and "experience with breastfeeding". In addition, 15 participants were unaware of the postnatal service provided by MCHCs.

\section{Opinions on the impact of COVID-19 on breastfeeding}

For participants whose child was twelve months or younger and whose workplace had adopted workfrom-home policies during the COVID-19 pandemic $(n=251)$, being able to work from home $(75.7 \%)$, wanting to boost the child immunity through breastfeeding (70.5\%), leaving home less $(70.1 \%)$, having more flexible work schedules (57.4\%), and having better family support at home (21.1\%) were common reasons why participants felt more encouraged to breastfeed during the pandemic. Meanwhile, $14.7 \%$ of participants wanted to avoid breastfeeding or expressing breast milk in public premises, so as to reduce their children's chance of being infected during the pandemic.

\section{Discussion}

This study complemented the existing literature regarding the barriers and facilitators of EBF by identifying three factors associated with successful six-month EBF, namely giving birth in a public hospital, the self-rated impact of COVID-19, and the support from family and friends. Such factors could be targeted by policymakers for designing EBF interventions.

Our qualitative results indicated a high prevalence of breastfeeding problems in both public and private maternity wards, suggesting that neonatal support for breastfeeding in both public and private hospitals were generally insufficient and ineffective. In particular, giving birth in private hospitals was associated with a lower likelihood of successful six-month EBF, both in general and during the pandemic period; our qualitative results identified four potential reasons for such a phenomenon. First, the inappropriate recommendation of formula supplementation by nurses in private hospitals likely reinforced PIMS amongst mothers, hence discouraged them from in-hospital EBF (BFHI Step 6) and responsive breastfeeding (BFHI Step 8) [21]. As demonstrated in both our study and other international studies, PIMS is a major factor for discontinued breastfeeding and EBF cessation [22-24]. Moreover, while in-hospital EBF has been demonstrated to be positively associated with continued breastfeeding or EBF in both local and international studies, in-hospital formula supplementation is a risk factor for early breastfeeding cessation worldwide [25-28]. Second, compared to public hospitals, private hospitals were less 
supportive of early in-hospital breastfeeding initiation (BFHI Step 4), a finding consistently implied from both local and international studies [8, 21, 29]. Yet, early in-hospital breastfeeding initiation is a factor strongly positively associated with breastfeeding establishment, in-hospital EBF, and continued breastfeeding worldwide $[21,28,30]$. Third, private hospital staff tended to offer conflicting and incomprehensive breastfeeding advice to mothers. This is likely due to the relatively poor communication of hospital breastfeeding policies (BFHI Step 1) and insufficient staff training (BFHI Step 2) in private hospitals as compared to public hospitals $[8,21]$. Fourth, private hospitals are generally unsupportive of rooming-in practice (BFHI Step 7), a factor significantly positively associated with in-hospital EBF [21, 31]. The poor rooming-in practice of private hospitals, as compared to public hospitals which all practised rooming-in, was observed in another local survey [8]. Overall, our results suggested that private hospitals were less supportive of in-hospital EBF and less committed to the BFHI than public hospitals, although public hospitals experienced labour shortage and lack breastfeeding support in SCBUs and NICUs. Further investigation is needed to assess whether similar trends are observed internationally.

We also found the pandemic in Hong Kong to have a generally positive self-rated impact on breastfeeding, which was positively associated with successful six-month EBF. Our qualitative results indicated that this is likely due to a combination of having work-from-home arrangements, more flexible work schedules, and leaving home less. Many mothers also hoped to protect their children from COVID-19 by transferring anti-infective factors and immune boosters through breast milk. In contrast, a study in Italy found lockdown and home confinement measures during COVID-19 to reduce EBF [16]. Such contrasting findings were likely due to two reasons. First, the more severe COVID-19 situation in Italy and uncertainties around vertical transmission of SARS-CoV-2 during the study period (March 2020 to Aug 2020) at the early stages of the pandemic made mothers and healthcare workers alike wary of breastfeeding [32,33]. Second, due to stringent lockdown restrictions in Italy that resulted in complete home confinement and reduced transportation, new families could not access breastfeeding support easily, including those from family and friends [34]. Meanwhile, the pandemic also presented unique difficulties to breastfeeding. A small proportion of mothers expressed worries regarding breastfeeding and expressing breast milk in public premises due to concerns of exposing the infant to possible infection. Moreover, the temporary suspension of postnatal services in MCHCs during pandemics could deprive mothers of critical breastfeeding support, hence negatively impact breastfeeding practice amongst mothers. Furthermore, during the pandemic, most antenatal classes and breastfeeding coaching services were cancelled, while some were moved online with the implementation of social distancing measures. Some participants further complained that online antenatal classes did not facilitate interactive teaching and effective learning; similar findings were observed in the UK [35].

Results of logistic regression models further indicated that breastfeeding support from family and friends was positively associated with successful six-month EBF, both in general and during the pandemic period. This is consistent with previous studies that emphasised the importance of the husband's preference for EBF and having peers who have had breastfeeding experience, as well as the positive impact of paternity leave on breastfeeding duration [36-39]. In the Chinese society, it is traditional for the grandparents to provide assistance to new mothers during the first month postpartum and as such, the 
attitude of grandparents strongly influences feeding practices [38]. The notion that EBF is not enough for the baby's satiety is strong amongst the older generation and reinforces PIMS in new mothers, a circumstance that often convinces new mothers to supplement with or switch to formula feeding $[40,41]$.

Both local and international studies have demonstrated that the exclusivity of breastfeeding and the duration of EBF are positively associated with maternal education level, breastfeeding knowledge, and household income [42-44]. However, due to our purposive sampling approach, our participants had a higher education level and socioeconomic status compared to the general population, and were more committed to breastfeeding [45]. As such, these associations were not observed in this study.

Strengths and limitations

Our study adopted a purposive sampling approach and targeted members of breastfeeding support groups and organisations, who were committed to breastfeeding, experienced with breastfeeding, and exposed to a wide range of breastfeeding services provided by different health care providers, organisations, and professionals. This allowed wide-ranging exploration on the barriers and facilitators of six-month EBF to help inform future research efforts. In addition, this is the first study undertaken to explore the impact of COVID-19 on breastfeeding practice in Hong Kong.

However, our study had some limitations. Due to the voluntary nature of this study, it was possible that those who participated had more positive attitudes towards breastfeeding, subjecting it to volunteer response bias. In addition, the purposive sampling approach limited the generalisability of our findings. Moreover, the deductive and inductive methods adopted in our qualitative analysis could oversimplify the individual breastfeeding experience of participants. Furthermore, self-reporting responses of participants were subject to recall bias. Lastly, responses regarding participants' perceived helpfulness of different health organisations and professionals might be influenced by the reduced accessibility of antenatal classes and MCHC services during the COVID-19 pandemic.

\section{Recommendations}

In view of our findings, we propose six areas which the government and hospitals should address to further promote in-hospital and six-month EBF in Hong Kong.

First, staff training in private hospitals should be strengthened. Written notice of breastfeeding policies should be well-communicated to all staff (BFHI Step 1), while standard training should be mandated for staff (BFHI Step 2), especially for nurses and midwives in private maternity wards [21].

Second, a postpartum home-based programme should be implemented, as recommended by other local studies $[17,46]$. Although our qualitative results suggested that practical, comprehensive, extensive, and interactive antenatal classes were welcomed by the mothers, the evenly divided rating of participants suggested that the helpfulness of existing antenatal classes was controversial. Results from other highincome countries consistently suggest that the efficacy of antenatal classes is uncertain [47]. In addition, mothers in Hong Kong may find it difficult to leave home during the early postpartum period due to the 
overwhelming workload and the cultural practice of "doing-the-month" [17]. Hence, the practical challenges of breastfeeding should be addressed postnatally in a home setting.

Third, breastfeeding support in SCBUs and NICUs of public hospitals should be strengthened. SCBUs and NICUs of public hospitals should offer clear breastfeeding guidance and support milk pumping, bottlefeeding, and breastfeeding as appropriate.

Fourth, $\mathrm{MCHC}$ services should be continued during pandemics and be further promoted on discharge. The provision of information regarding breastfeeding support on discharge (BFHI Step 10) was associated with continued breastfeeding both locally and internationally [21, 26, 28]. While most public and private hospitals in Hong Kong self-reported that they provided such support, our qualitative results indicated that some participants were unaware of such services [8], suggesting a communication gap which should be addressed through active promotion.

Fifth, the spouse and the grandparents should be included in breastfeeding education and antenatal classes. Since family members play a significant role in the choice and duration of EBF, it is important to cultivate their knowledge on breastfeeding through family-centred breastfeeding education $[48,49]$.

Sixth, flexible work-from-home arrangements should be provided to parents following the end of existing parental leaves. Our qualitative results suggested that returning to work from maternity leave is a major reason for EBF cessation, while other studies indicated that parents' return to work was a significant predictor of early weaning $[39,46,50]$. Yet, the recent extension of statutory maternity leave to 14 weeks in Hong Kong still falls short of the six-month target, while statutory paternity leave lasts for five days only $[7,51]$. With the experience of large-scale work-from-home arrangements during COVID-19, similar arrangements could be considered as an alternative to extending existing parental leaves.

\section{Conclusions}

This study identified the positive factors associated with six-month EBF in Hong Kong women, namely giving birth in a public hospital and breastfeeding support from family and friends. Furthermore, the COVID-19 pandemic in Hong Kong had an overall positive impact on six-month EBF. Further studies should investigate the impact of hospital practice and the COVID-19 pandemic on breastfeeding behaviours.

\section{Abbreviations}

AOR

Adjusted odds ratio

BFHI

Baby-Friendly Hospital Initiative

BFHIHKA

Baby Friendly Hospital Initiative Hong Kong Association 
$\mathrm{Cl}$

Confidence interval

EBF

Exclusive breastfeeding

EFA

Exploratory factor analysis

HKBFMA

Hong Kong Breastfeeding Mothers' Association

IBCLC

International Board Certified Lactation Consultant

LLLHK

La Leche League Hong Kong

$\mathrm{MCHC}$

Maternal and Child Health Centre

NICU

Neonatal intensive care unit

PIMS

Perceived insufficient milk supply

SCBU

Special care baby unit

Six-month EBF

The practice of exclusive breastfeeding for at least six months since birth

UNICEF

United Nations Children's Fund

WHO

World Health Organization

\section{Declarations}

\section{Ethics approval and consent to participate}

Ethical approval was obtained from the Institutional Review Board (IRB) of the University of Hong Kong/Hospital Authority Hong Kong West Cluster (IRB Reference Number: UW 21-180). Informed consent was obtained from all the participants before data collection.

\section{Consent for publication}

Not applicable. 


\section{Availability of data and materials}

The datasets used and analysed during this study are available from the corresponding author on reasonable request.

\section{Competing interests}

The authors declare that they have no competing interests.

\section{Funding}

Not applicable.

\section{Authors' contributions}

JK and JJ developed the first draft. KY, HS, SSFL, PI, and WHSW conceptualised the manuscript. All authors read, revised, and approved the final manuscript for publication.

\section{Acknowledgements}

The authors would like to express our special thanks to everyone who participated in this study, including the members from Hong Kong Breastfeeding Mothers' Association (HKBFMA) and La Leche League Hong Kong (LLLHK). We are indebted to the panel members including Jacy Chan, Stephanie Lam, Lydia Ling, Michelle Lok, and Crystie Wong for the questionnaire design.

\section{References}

1. World Health Organization. Exclusive breastfeeding for optimal growth, development and health of infants. https://www.who.int/elena/titles/exclusive_breastfeeding/en/. Accessed 27 June 2021.

2. United Nations Children's Fund. Scientific rationale: benefits of breastfeeding. https://www.unicef.org/Scientific_rationale_for_benefits_of_breasfteeding.pdf. Accessed 27 June 2021.

3. World Health Organization, United Nations Children's Fund. Global breastfeeding scorecard. 2019: increasing commitment to breastfeeding through funding and improved policies and programmes. World Health Organization; 2019. No.: WHO/NMH/NHD/19.22.

4. Tarrant M. Submission to the Legislative Council Panel on Food Safety and Environmental Hygiene and Panel on Health Services on the Hong Kong Code of Marketing and Quality of Formula Milk and 
Related Products and Food Products for Infants and Young Children. HK: School of Nursing, The University of Hong Kong; 2012. No.: CB(2)237/12-13(02).

5. Hong Kong Code of Marketing of Formula Milk and Related Products. and Food Products for Infants \& Young Children (HK).

6. Discrimination Legislation. (Miscellaneous Amendments) Ordinance 2020 (HK).

7. The Employment. (Amendment) Ordinance 2020 (HK).

8. Baby Friendly Hospital Initiative Hong Kong Association. World Breastfeeding Week (WBW) 1-7 August 2020. HK: Baby Friendly Hospital Initiative Hong Kong Association; 2020.

9. Lee SY. The 2018 National Survey on Fertility and Family Health and Welfare. KR: Institute for Health and Social Affairs; 2018. No.: 2018-37.

10. Family Health Service. Breastfeeding Survey 2019. HK: Department of Health; 2019.

11. Inano H, Kameya M, Sasano K, Matsumura K, Tsuchida A, Hamazaki K, et al. Factors influencing exclusive breastfeeding rates until 6 months postpartum: the Japan Environment and Children's Study. Sci Rep. 2021;11(1):6841. http://dx.doi.org/10.1038/s41598-021-85900-4.

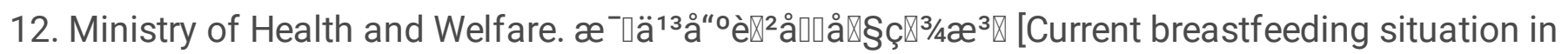
Taiwan]. https://www.hpa.gov.tw/Pages/Detail.aspx?nodeid=506\&pid=463. Accessed 27 June 2021. Chinese.

13. Office of the Surgeon General. Barriers to breastfeeding in the United States. Rockville: Office of the Surgeon General; 2011.

14. News.gov.hk. Work from home plan advised. https://www.news.gov.hk/eng/2020/07/20200727/20200727_180031_822.html. Accessed 27 June 2021.

15. Family Health Service. Family Health Service - Temporary closure of 5 Maternal and Child Health Centres (MCHCs) and re-arrangement of service. https://www.fhs.gov.hk/english/news/. Accessed 27 June 2021.

16. Latorre G, Martinelli D, Guida P, Masi E, De Benedictis R, Maggio L. Impact of COVID-19 pandemic lockdown on exclusive breastfeeding in non-infected mothers. Int Breastfeed J. 2021;16(1):36. http://dx.doi.org/10.1186/s13006-021-00382-4.

17. Tarrant M, Dodgson JE, Wu KM. Factors contributing to early breast-feeding cessation among Chinese mothers: an exploratory study. Midwifery. 2014;30(10):1088-95. http://dx.doi.org/10.1016/j.midw.2014.03.002.

18. Baby Friendly Hospital Initiative Hong Kong Association. Myths on breastfeeding_FAQ. https://www.babyfriendly.org.hk/en/myths-on-breastfeeding_faq-2/. Accessed 27 June 2021.

19. Family Health Service. Survey on mothers' breastfeeding experience in public places. HK: Department of Health; 2016.

20. Pituch KA, Stevens JP. Applied multivariate statistics for the social sciences: Analyses with SAS and IBM's SPSS. 6th ed.: Routledge; 2016. 
21. World Health Organization, United Nations Children's Fund. Implementation guidance: protecting, promoting and supporting breastfeeding in facilities providing maternity and newborn services: the revised baby-friendly hospital initiative. World Health Organization; 2018.

22. Gatti L. Maternal perceptions of insufficient milk supply in breastfeeding. J Nurs Scholarsh. 2008;40(4):355-63. http://dx.doi.org/10.1111/j.1547-5069.2008.00234.x.

23. Hauck YL, Fenwick J, Dhaliwal SS, Butt J. A Western Australian survey of breastfeeding initiation, prevalence and early cessation patterns. Matern Child Health J. 2011;15(2):260-8. http://dx.doi.org/10.1007/s10995-009-0554-2.

24. Galipeau R, Baillot A, Trottier A, Lemire L. Effectiveness of interventions on breastfeeding selfefficacy and perceived insufficient milk supply: A systematic review and meta-analysis. Matern Child Nutr. 2018;14(3):e12607. http://dx.doi.org/10.1111/mcn.12607.

25. Tarrant M, Wu KM, Fong DY, Lee IL, Wong EM, Sham A, et al. Impact of baby-friendly hospital practices on breastfeeding in Hong Kong. Birth. 2011;38(3):238-45. http://dx.doi.org/10.1111/j.1523-536X.2011.00483.x.

26. Lok KYW, Chow CLY, Fan HSL, Chan VHS, Tarrant M. Exposure to baby-friendly hospital practices and mothers' achievement of their planned duration of breastfeeding. BMC Pregnancy Childbirth. 2020;20(1):261. http://dx.doi.org/10.1186/s12884-020-02904-0.

27. Cascone D, Tomassoni D, Napolitano F, Di Giuseppe G. Evaluation of Knowledge, Attitudes, and Practices about Exclusive Breastfeeding among Women in Italy. Int J Environ Res Public Health. 2019;16(12). http://dx.doi.org/10.3390/ijerph16122118.

28. Pérez-Escamilla R, Martinez JL, Segura-Pérez S. Impact of the Baby-friendly Hospital Initiative on breastfeeding and child health outcomes: a systematic review. Matern Child Nutr. 2016;12(3):40217. http://dx.doi.org/10.1111/mcn.12294.

29. Oakley L, Benova L, Macleod D, Lynch CA, Campbell OMR. Early breastfeeding practices: Descriptive analysis of recent Demographic and Health Surveys. Matern Child Nutr. 2018;14(2):e12535. http://dx.doi.org/10.1111/mcn.12535.

30. World Health Organization. United Nations Children's Fund. Capture the moment - Early initiation of breastfeeding: the best start for every newborn. World Health Organization; 2018.

31. Jaafar SH, Ho JJ, Lee KS. Rooming-in for new mother and infant versus separate care for increasing the duration of breastfeeding. Cochrane Database Syst Rev. 2016(8):Cd006641. http://dx.doi.org/10.1002/14651858.CD006641.pub3.

32. Bastug A, Hanifehnezhad A, Tayman C, Ozkul A, Ozbay O, Kazancioglu S, et al. Virolactia in an Asymptomatic Mother with COVID-19. Breastfeed Med. 2020;15(8):488-91. http://dx.doi.org/10.1089/bfm.2020.0161.

33. Our World in Data. Italy: Coronavirus pandemic country profile. https://ourworldindata.org/coronavirus/country/italy. Accessed 27 June 2021.

34. Remuzzi A, Remuzzi G. COVID-19 and Italy: what next? Lancet. 2020;395(10231):1225-8. http://dx.doi.org/10.1016/s0140-6736(20)30627-9. 
35. Nolan M. Educators' experience of facilitating antenatal education online. IJBPE. 2021.

36. Kong SK, Lee DT. Factors influencing decision to breastfeed. J Adv Nurs. 2004;46(4):369-79. http://dx.doi.org/10.1111/j.1365-2648.2004.03003.x.

37. Lok KYW, Bai DL, Tarrant M. Family members' infant feeding preferences, maternal breastfeeding exposures and exclusive breastfeeding intentions. Midwifery. 2017;53:49-54.

http://dx.doi.org/10.1016/j.midw.2017.07.003.

38. Bai DL, Fong DY, Lok KY, Tarrant M. Relationship between the infant feeding preferences of Chinese mothers' immediate social network and early breastfeeding cessation. J Hum Lact. 2016;32(2):3018. http://dx.doi.org/10.1177/0890334416630537.

39. Flacking R, Dykes F, Ewald U. The influence of fathers' socioeconomic status and paternity leave on breastfeeding duration: a population-based cohort study. Scand J Public Health. 2010;38(4):337-43. http://dx.doi.org/10.1177/1403494810362002.

40. Tarrant M, Dodgson JE, Tsang Fei S. Initiating and sustaining breastfeeding in Hong Kong: contextual influences on new mothers' experiences. Nurs Health Sci. 2002;4(4):181-91. http://dx.doi.org/10.1046/j.1442-2018.2002.00125.x.

41. Zhang K, Tang L, Wang H, Qiu L, Binns CW, Lee AH. Why do mothers of young infants choose to formula feed in China? Perceptions of mothers and hospital staff. Int J Environ Res Public Health. 2015;12(5):4520-32. http://dx.doi.org/10.3390/ijerph120504520.

42. Ku CM, Chow SK. Factors influencing the practice of exclusive breastfeeding among Hong Kong Chinese women: a questionnaire survey. J Clin Nurs. 2010;19(17-18):2434-45. http://dx.doi.org/10.1111/j.1365-2702.2010.03302.x.

43. Zielińska MA, Sobczak A, Hamułka J. Breastfeeding knowledge and exclusive breastfeeding of infants in first six months of life. Rocz Panstw Zakl Hig. 2017;68(1):51-9.

44. Peregrino AB, Watt RG, Heilmann A, Jivraj S. Breastfeeding practices in the United Kingdom: Is the neighbourhood context important? Matern Child Nutr. 2018;14(4):e12626. http://dx.doi.org/10.1111/mcn.12626.

45. Census and Statistics Department. 2016 Population By-census. https://www.censtatd.gov.hk/en/scode459.html. Accessed 27 June 2021.

46. Tarrant M, Fong DY, Wu KM, Lee IL, Wong EM, Sham A, et al. Breastfeeding and weaning practices among Hong Kong mothers: a prospective study. BMC Pregnancy Childbirth. 2010;10:27. http://dx.doi.org/10.1186/1471-2393-10-27.

47. Lumbiganon P, Martis R, Laopaiboon M, Festin MR, Ho JJ, Hakimi M. Antenatal breastfeeding education for increasing breastfeeding duration. Cochrane Database Syst Rev. 2016;12(12):Cd006425. http://dx.doi.org/10.1002/14651858.CD006425.pub4.

48. Su M, Ouyang YQ. Father's role in breastfeeding promotion: Lessons from a quasi-experimental trial in China. Breastfeed Med. 2016;11:144-9. http://dx.doi.org/10.1089/bfm.2015.0144.

49. Susin LR, Giugliani ER. Inclusion of fathers in an intervention to promote breastfeeding: impact on breastfeeding rates. J Hum Lact. 2008;24(4):386-92. 
http://dx.doi.org/10.1177/0890334408323545. quiz 451-3.

50. Stuebe AM, Horton BJ, Chetwynd E, Watkins S, Grewen K, Meltzer-Brody S. Prevalence and risk factors for early, undesired weaning attributed to lactation dysfunction. J Womens Health (Larchmt). 2014;23(5):404-12. http://dx.doi.org/10.1089/jwh.2013.4506.

51. The Employment Ordinance. Cap. 57 (HK).

\section{Supplementary Files}

This is a list of supplementary files associated with this preprint. Click to download.

- Additionalfile1.pdf 